\title{
Curvature pinching estimate and singularities of the Ricci flow
}

\author{
XiaOdong CAO
}

\begin{abstract}
In this paper, we first derive a pinching estimate on the traceless Ricci curvature in term of scalar curvature and Weyl tensor under the Ricci flow. This generalizes a previous result of Knopf [15]. Then we apply this estimate to study finite-time singularity behavior. We show that if the scalar curvature is uniformly bounded, then the Weyl tensor has to blow up at least at a certain rate.
\end{abstract}

\section{Introduction}

Let $(M, g)$ be a smooth, closed $n$-dimensional Riemannian manifold. In his seminal paper [8], Hamilton proved that any closed three-manifold which admits a Riemannian metric with strictly positive Ricci curvature must also admit a metric of constant positive sectional curvature. He showed that the original metric can be deformed into the constant-curvature metric by introducing the Ricci flow:

$$
\frac{\partial}{\partial t} g_{i j}=-2 R_{i j}
$$

The Ricci flow equation is a (weakly) parabolic partial differential equation system. Its short time existence was first proved by Hamilton [8] and later the proof was simplified by DeTurck [6]. One of the main subjects in the study of Ricci flow is the understanding of long time behavior and formation of singularities. More precisely, we would like to ask when the flow can exist for all time; and if the flow only exits up to finite time, we would like to understand the profile of finite-time singularities, which in general will permit us to understand geometry and topology of the underlying manifold better.

A solution $(M, g(t))$ to the Ricci flow equation (1.1) is a (finite-time) maximal solution if it is defined for $t \in[0, T), T<\infty$. In [8], Hamilton proved that the whole Riemannian curvature tensor Rm blows up as $t \rightarrow$ $T$, i.e., $\lim \sup _{[0, T)}|\mathrm{Rm}|=\infty$. In [25], Sesum showed that in fact the Ricci curvature tensor Rc blows up as $t \rightarrow T$, i.e., $\lim \sup _{[0, T)}|\operatorname{Rc}|=\infty$. In other 
words, if the norm of Riemannian curvature or Ricci curvature is uniformly bounded on $[0, T)$, then the flow can be smoothly extended past $T$. In [27], Wang extended the above results even further by showing that if the Ricci curvature tensor $\mathrm{Rc}$ is uniformly bounded from below and moreover, the space-time integral of scalar curvature $R$ is bounded, namely,

$$
\int_{0}^{T} \int_{M}|R|^{\alpha} \leq C, \alpha \geq \frac{n+2}{2}
$$

then the Ricci flow can be smoothly extended past $T$. Similar type results also appeared in [29] by Ye and in [16] by Ma and Cheng.

There is a well-known conjecture that the scalar curvature $R$ should also blow up at the finite singular time $T$. Recently, Enders et al. [7] partially confirmed this conjecture in the case of Type I maximal solutions. They used blow-up argument based on Perelman's reduced distance and pseudolocality theorem. In the case of Kähler-Ricci flow, this was proved by Zhang [30].

Definition 1.1. A maximal solution $(M, g(t)), 0 \leq t<T<\infty$, is called a Type I maximal solution of the Ricci flow, if there exists a constant $C<\infty$ such that the curvature satisfies

$$
|\mathrm{Rm}| \leq \frac{C}{T-t} .
$$

Otherwise it is a Type II maximal solution of the Ricci flow.

In this paper, we study the blow-up behavior of different components of the curvature tensor under the Ricci flow, and their consequences in dilation limits.

For simplicity, we also use the following convention: the constants $c_{i}$ only depend on the dimension $n$, but not on the initial metric $g(0)$; while the constants $C_{i}$ depend not only on the dimension $n$, but also on the initial metric $g(0)$. We also restrict ourselves to the case of positive scalar curvature, even though that most estimates in this paper can be carried to the general case.

The rest of this paper organized as follows. In Section 2, we briefly review the orthogonal decomposition of Riemannian curvature and evolution of curvatures under the Ricci flow. In Section 3, we derive a pinching estimate on the traceless Ricci curvature tensor, which generalizes a previous result of Knopf [15]. As one application, we obtain some information about curvature blow up at finite-time; the second application is on manifolds with positive 
isotropic curvature. In Section 4, we discuss singularity models and apply the pinching estimate in Section 3 to study the dilation limit.

\section{Decomposition and evolution of curvature tensors}

In this section, we will first give a brief introduction of curvature decomposition of the Riemannian manifold $\left(M^{n}, g\right)$ and some relations of geometric conditions. Then we will recall some evolution formulae for various curvature tensors, for more details, please see [8]. We use $g_{i j}$ to denote the local components of metric $g$ and its inverse by $g^{i j}$. In this paper, we use Rm to denote the $(4,0)$ Riemannian curvature tensor instead of the $(3,1)$ Riemannian curvature tensor, we denote its local components by $R_{i j k l}$. Let Rc be the Ricci curvature with local components $R_{i k}=g^{j l} R_{i j k l}$, and let $R=g^{i k} R_{i k}$ be the scalar curvature. We first recall the Kulkarni-Nomizu product for two symmetric two-tensors $h, k$ is defined as

$$
\begin{aligned}
h \circ k\left(v_{1}, v_{2}, v_{3}, v_{4}\right)= & h\left(v_{1}, v_{3}\right) k\left(v_{2}, v_{4}\right)+h\left(v_{2}, v_{4}\right) k\left(v_{1}, v_{3}\right) \\
& -h\left(v_{1}, v_{4}\right) k\left(v_{2}, v_{3}\right)-h\left(v_{2}, v_{3}\right) k\left(v_{1}, v_{4}\right) .
\end{aligned}
$$

The Einstein tensor or traceless Ricci tensor $E$ is defined as

$$
E_{i j}=R_{i j}-\frac{R}{n} g_{i j}
$$

When $n \geq 4$, we can decompose the $(4,0)$ Riemannian curvature tensor Rm in the following way:

$$
\mathrm{Rm}=\frac{R}{2 n(n-1)} g \circ g+\frac{1}{n-2} E \circ g+W,
$$

where $W$ is the Weyl curvature tensor. And the above decompositions are orthogonal.

In local coordinates, we can write

$$
\begin{aligned}
W_{i j k l}= & R_{i j k l}-\frac{1}{n-2}\left(g_{i k} R_{j l}+g_{j l} R_{i k}-g_{i l} R_{j k}-g_{j k} R_{i l}\right) \\
& +\frac{1}{(n-1)(n-2)} R\left(g_{i k} g_{j l}-g_{i l} g_{j k}\right) .
\end{aligned}
$$

It is well known that under conformal change of the metric $g^{\prime}=\mathrm{e}^{u} \cdot g$ for some function $u$, then $W^{\prime}=\mathrm{e}^{u} \cdot W$. If we view the Weyl tensor as a $(3,1)$ tensor, then $W^{\prime}=W$, i.e., the $(3,1)$ Weyl tensor is a conformal invariant. 
Under the Ricci flow, the Ricci curvature is evolving by

$$
\frac{\partial}{\partial t} \mathrm{Rc}=\triangle \mathrm{Rc}+2 \mathrm{Rm}(\mathrm{Rc}, \cdot)-2 \mathrm{Rc}^{2}
$$

where $\mathrm{Rc}_{i j}^{2}=R_{i k} R_{k j}$ and the scalar curvature evolves by

$$
\frac{\partial}{\partial t} R=\triangle R+2|\mathrm{Rc}|^{2}
$$

As a direct consequence of (2.1), in all dimensions, the positivity (or any lower bound) of the scalar curvature is preserved by the Ricci flow. In dimension 3, the positivity of Ricci curvature is preserved (see [8]). In dimension at least 4 , positivity of curvature operator is preserved $[9,11]$.

In [17], Micallef and Moore introduced a new curvature condition, positive isotropic curvature. A Riemannian manifold of dimension at least 4 is said to have positive isotropic curvature, if for every orthonormal four-frame $\left\{e_{1}, e_{2}, e_{3}, e_{4}\right\}$, we have

$$
R_{1313}+R_{1414}+R_{2323}+R_{2424}-2 R_{1234}>0
$$

Using minimal surface technique, they proved that any compact, simply connected manifold with positive isotropic curvature is homeomorphic to $S^{n}$. In the same paper, they observed that the positivity of isotropic curvature is implied by several other commonly used curvature conditions, such as positive curvature operator and pointwise $\frac{1}{4}$-pinched condition. In dimension 4, Hamilton [12] proved that the positivity of isotropic curvature is preserved by the Ricci flow. This result has been extended to higher dimensions by Brendle and Schoen [2] and also by Nguyen [19] independently. Brendle and Schoen further proved the differentiable sphere theorem, which has been a long-time conjecture since the (topological) $\frac{1}{4}$-pinched sphere theorem proved by Berger [1] and Klingenberg [14] around 1960. More precisely, Brendle and Schoen showed that any compact Riemannian manifold with pointwise $\frac{1}{4}$-pinched sectional curvature is diffeomorphic to a spherical space form [2].

Another interesting geometric operator in Riemannian geometry, the Weitzenböck operator $P$, is defined as

$$
P=\operatorname{Rc} \circ g-2 \mathrm{Rm}=\frac{(n-2) R}{n(n-1)} g \circ g+\frac{n-4}{n-2} E \circ g-W,
$$


or in local coordinates,

$$
P_{i j k l}=\left(g_{i k} R_{j l}+g_{j l} R_{i k}-g_{i l} R_{j k}-g_{j k} R_{i l}\right)-2 R_{i j k l} .
$$

It is known that in dimension 4, positive isotropic curvature is equivalent to positive Weitzenböck operator (see for example, [17, 18, 20, 21]). For an even dimensional Riemannian manifold of $n>4$, positive isotropic curvature implies positive Weitzenböck operator [24, Proposition 1.1].

\section{Curvature pinching estimate}

The general evolution formulae of curvature tensors suggests that the orthogonal parts of Riemannian curvature tensor is not evolving totally independently to each other, one part might depend on the other part(s). An interesting question in the study of the Ricci flow is which orthogonal part(s) needs to blow up at a finite-time $T$ when singularity occurs. In other words, if these parts are uniformly bounded up to time $T$, then the Ricci flow can be smoothly extended past $T$.

Our main theorem in this section is the following estimate, which says that the traceless Ricci part $|E|$ can be controlled by the scalar curvature $R$ and Weyl tensor $|W|$. This improves an earlier result of Knopf [15].

Theorem 3.1. Let $\left(M^{n}, g(t)\right), t \in[0, T)$, be a solution to the Ricci flow on a closed Riemannian manifold of dimension $n \geq 3$, then there exist constants $C_{1}\left(n, g_{0}\right)>0, c_{2}(n) \geq 0$ and $c\left(n, g_{0}\right) \geq 0$, such that for all $t \geq 0$, one has $R+c>0$ and

$$
\frac{|E|}{R+c} \leq C_{1}+c_{2} \max _{M \times[0, t]} \sqrt{\frac{|W|}{R+c}}
$$

Furthermore, if $R>0$ at $t=0$, then we have

$$
\frac{|E|}{R} \leq C_{1}+c_{2} \max _{M \times[0, t]} \sqrt{\frac{|W|}{R}} .
$$

Remark 3.1. In [15], Knopf first proved a rather surprising result. Namely he showed that under the Ricci flow, there exist constants $c\left(g_{0}\right) \geq 0, C_{1}(n$, $\left.g_{0}\right)>0$ and $c_{2}(n)>0$ such that for all $t \geq 0$, one has $R+c>0$ and

$$
\frac{|E|}{R+c} \leq C_{1}+c_{2} \max _{s \in[0, t]} \sqrt{\frac{|W|_{\max }(s)}{R_{\min }(s)+c}} .
$$


In other words, the traceless Ricci part $|E|$ can be controlled by the maximum and minimum of the scalar curvature $R$ and the maximum of the Weyl tensor $|W|$. Notice that $|W|_{\max }$ and $R_{\min }$ may actually be achieved at different space-time points in (3.3). In estimates (3.1) and (3.2), the values of $|W|$ and $R$ are taken at the same point, this allows us to study dilation limits of singularities. In some cases, it will also allow us to study the blowup rate of singularities.

Remark 3.2. We state the theorem both for the general case and for the positive scalar curvature case. But for simplicity, we will only prove (3.2) here, and the proof of (3.1) is similar.

Remark 3.3. The estimate (3.2) is scaling invariant, so it still holds for normalized Ricci flow and also for Ricci flow solutions exist for all time $[0, \infty)$. In the special case of Kähler-Ricci flow on a compact Kähler manifold $X$ with $c_{1}(X)>0$, it is shown by Cao [3] that the solution exists for all time. It is also known that the scalar curvature is uniformly bounded along the flow (a detailed proof is given by Sesum and Tian [26] following Perelman's idea), hence the whole curvature tensor blows up if and only if the Weyl tensor $W$ blows up (also see [16]).

For our purpose, we perform a rather general calculation here. For any positive number $\gamma$, define

$$
f=\frac{|E|^{2}}{R^{\gamma}}=\frac{|\mathrm{Rc}|^{2}}{R^{\gamma}}-\frac{1}{n} R^{2-\gamma},
$$

then $f$ satisfies the following evolution equation.

Lemma 3.1. Under the Ricci flow, we have

$$
\begin{aligned}
\frac{\partial}{\partial t} f= & \triangle f+\frac{2(\gamma-1)}{R} \nabla f \cdot \nabla R-\frac{2}{R^{2+\gamma}}\left|R \nabla_{i} R_{j k}-\nabla_{i} R R_{j k}\right|^{2} \\
& -\frac{(2-\gamma)(\gamma-1)}{R^{2}}|\nabla R|^{2} f-\frac{2(2-\gamma)}{n} R^{1-\gamma}|\mathrm{Rc}|^{2} \\
& +\frac{4}{R^{\gamma}} \operatorname{Rm}(\mathrm{Rc}, \mathrm{Rc})-\frac{2 \gamma}{R^{1+\gamma}}|\mathrm{Rc}|^{4}-\frac{(2-\gamma)(\gamma-1)}{n R^{\gamma}} \\
= & \triangle f+\frac{2(\gamma-1)}{R} \nabla f \cdot \nabla R-\frac{2}{R^{2+\gamma}}\left|R \nabla_{i} R_{j k}-\nabla_{i} R R_{j k}\right|^{2}
\end{aligned}
$$


Curvature pinching estimate and singularities of the Ricci flow 981

$$
\begin{aligned}
& -\frac{(2-\gamma)(\gamma-1)}{R^{2}}|\nabla R|^{2} f+\frac{2}{R^{1+\gamma}}\left[(2-\gamma)|\mathrm{Rc}|^{2}\left(|\mathrm{Rc}|^{2}-\frac{1}{n} R^{2}\right)\right. \\
& -2\left(|\mathrm{Rc}|^{4}-R \cdot \operatorname{Rm}(\mathrm{Rc}, \mathrm{Rc})\right]-\frac{(2-\gamma)(\gamma-1)}{n R^{\gamma}} .
\end{aligned}
$$

Proof. We have

$$
\frac{\partial}{\partial t}|\mathrm{Rc}|^{2}=\triangle|\mathrm{Rc}|^{2}-2|\nabla \mathrm{Rc}|^{2}+4 \mathrm{Rm}(\mathrm{Rc}, \mathrm{Rc}),
$$

where

$$
\operatorname{Rm}(\mathrm{Rc}, \mathrm{Rc})=R_{a b c d} R_{a c} R_{b d} .
$$

We can further express the term as

$$
\begin{aligned}
\operatorname{Rm}(\mathrm{Rc}, \mathrm{Rc})= & R_{a b c d} R_{a c} R_{b d}=\frac{1}{n-2}\left(\frac{2 n-1}{n-1}|\mathrm{Rc}|^{2} R-2 \mathrm{Rc}^{3}-\frac{R^{3}}{n-1}\right) \\
& +W(\mathrm{Rc}, \mathrm{Rc}),
\end{aligned}
$$

hence we arrive at,

$$
\begin{aligned}
\frac{\partial}{\partial t}|\mathrm{Rc}|^{2}= & \triangle|\mathrm{Rc}|^{2}-2|\nabla \mathrm{Rc}|^{2}+\frac{4}{n-2}\left(\frac{2 n-1}{n-1}|\mathrm{Rc}|^{2} R-2 \mathrm{Rc}^{3}-\frac{R^{3}}{n-1}\right) \\
& +W(\mathrm{Rc}, \mathrm{Rc}) .
\end{aligned}
$$

Using this together with the evolution equation of the scalar curvature

$$
\frac{\partial}{\partial t} R=\triangle R+2|\mathrm{Rc}|^{2}
$$

we have the following two equations:

$$
\begin{aligned}
\frac{\partial}{\partial t}\left(\frac{|\mathrm{Rc}|^{2}}{R^{\gamma}}\right)= & \triangle\left(\frac{|\mathrm{Rc}|^{2}}{R^{\gamma}}\right)+\frac{2(\gamma-1)}{R} \nabla\left(\frac{|\mathrm{Rc}|^{2}}{R^{\gamma}}\right) \cdot \nabla R \\
& -\frac{2}{R^{2+\gamma}}\left|R \nabla_{i} R_{j k}-\nabla_{i} R R_{j k}\right|^{2}-\frac{(2-\gamma)(\gamma-1)}{R^{2+\gamma}}|\mathrm{Rc}|^{2}|\nabla R|^{2} \\
& +\frac{4}{R^{\gamma}} \operatorname{Rm}(\mathrm{Rc}, \mathrm{Rc})-\frac{2 \gamma}{R^{1+\gamma}}|\mathrm{Rc}|^{4},
\end{aligned}
$$

and

$$
\frac{\partial}{\partial t} R^{2-\gamma}=\triangle R^{2-\gamma}+\frac{2(\gamma-1)}{R} \nabla R^{2-\gamma} \cdot \nabla R+2(2-\gamma) R^{1-\gamma}|\mathrm{Rc}|^{2} .
$$

The lemma then follows. 
We can also rewrite the above lemma in the following way.

Lemma 3.2. Under the Ricci flow, we have

$$
\begin{aligned}
\frac{\partial}{\partial t} f= & \triangle f+\frac{2(\gamma-1)}{R} \nabla f \cdot \nabla R-\frac{2}{R^{2+\gamma}}\left|R \nabla_{i} R_{j k}-\nabla_{i} R R_{j k}\right|^{2} \\
& -\frac{(2-\gamma)(\gamma-1)}{R^{2}}|\nabla R|^{2} f+\frac{2}{R^{1+\gamma}}\left[(2-\gamma)|\mathrm{Rc}|^{2}|E|^{2}\right. \\
& -2 Q+2 R W(\mathrm{Rc}, \mathrm{Rc})]-\frac{(2-\gamma)(\gamma-1)}{n R^{\gamma}} \\
= & \triangle f+\frac{2(\gamma-1)}{R} \nabla f \cdot \nabla R-\frac{2}{R^{2+\gamma}}\left|R \nabla_{i} R_{j k}-\nabla_{i} R R_{j k}\right|^{2} \\
& -\frac{(2-\gamma)(\gamma-1)}{R^{2}}|\nabla R|^{2} f+\frac{2}{R^{1+\gamma}} \\
& \times\left[-\gamma|E|^{4}+\left(\frac{2(n-2)}{n(n-1)}-\frac{\gamma}{n}\right)|R|^{2}|E|^{2}-\frac{4}{n-2} R E^{3}+2 R W(E, E)\right] \\
& -\frac{(2-\gamma)(\gamma-1)}{n R^{\gamma}},
\end{aligned}
$$

where $Q=|\mathrm{Rc}|^{4}-\frac{R}{n-2}\left(\frac{2 n-1}{n-1} R|\mathrm{Rc}|^{2}-2 \mathrm{Rc}^{3}-\frac{R^{3}}{n-1}\right)$, and $E^{3}=E_{i j} E_{j k} E_{k i}$.

Consider the special case that $\gamma=2$, i.e.,

$$
f=\frac{|E|^{2}}{R^{2}}=\frac{|\mathrm{Rc}|^{2}}{R^{2}}-\frac{1}{n}
$$

we have

Lemma 3.3. Under the Ricci flow, we have

$$
\begin{aligned}
\frac{\partial}{\partial t} f= & \Delta f+\frac{2}{R} \nabla f \cdot \nabla R-\frac{2}{R^{4}}\left|R \nabla_{i} R_{j k}-\nabla_{i} R R_{j k}\right|^{2} \\
& +\frac{2}{R^{3}}\left[-2|E|^{4}-\frac{2}{n(n-1)}|R|^{2}|E|^{2}-\frac{4}{n-2} R E^{3}+2 R W(E, E)\right] \\
= & \Delta f+\frac{2}{R} \nabla f \cdot \nabla R-\frac{2}{R^{4}}\left|R \nabla_{i} R_{j k}-\nabla_{i} R R_{j k}\right|^{2} \\
& +4 R\left[-f^{2}-\frac{1}{n(n-1)} f-\frac{2}{n-2} \frac{E^{3}}{R^{3}}+\frac{1}{R^{3}} W(E, E)\right] .
\end{aligned}
$$


To estimate the right-hand side of the above equation, we claim that there exist positive constants $c_{1}, c_{2}$ depending only on $n \geq 3$, such that

$$
\left|\frac{2}{n-2} E^{3}\right| \leq c_{1}|E|^{3}
$$

and

$$
|W(E, E)| \leq c_{2}|W||E|^{2} .
$$

Remark 3.4. In the above estimates, $c_{1} \sim \frac{2}{n(n-2)}$ and $c_{2} \sim n(n-1)$ $(n-2)(n-3)$.

Plugging these two inequalities into Lemma 3.3, we derive that

Lemma 3.4. Under the Ricci flow, we have

$$
\begin{aligned}
\frac{\partial}{\partial t} f & \leq \triangle f+\frac{2}{R} \nabla f \cdot \nabla R+4 R\left[-f^{2}-\frac{1}{n(n-1)} f+c_{1} f^{3 / 2}+c_{2} \frac{|W|}{R} f\right] \\
& =\triangle f+\frac{2}{R} \nabla f \cdot \nabla R-4 R f\left[f-c_{1} f^{1 / 2}+\frac{1}{n(n-1)}-c_{2} \frac{|W|}{R}\right] .
\end{aligned}
$$

Combining the above inequality and using maximum principle, this leads to the following

Lemma 3.5. Under the Ricci flow, there exists $C_{1}=C_{1}\left(c_{1}, g(0)\right) \geq c_{1}>0$, such that $4 f(0) \leq C_{1}^{2}$. Moreover, we have

$$
f^{1 / 2} \leq \frac{1}{2} C_{1}+\sqrt{\frac{1}{4} C_{1}^{2}-\left(\frac{1}{n(n-1)}-c_{2} \max _{M \times[0, t]} \frac{|W|}{R}\right)} .
$$

Proof. Let us denote the right side as $\Phi(t)$, i.e.,

$$
\Phi(t)=\frac{1}{2} C_{1}+\sqrt{\frac{1}{4} C_{1}^{2}-\left(\frac{1}{n(n-1)}-c_{2} \max _{M \times[0, t]} \frac{|W|}{R}\right)},
$$

so $\Phi(t)$ is nondecreasing, by our choice of $C_{1}, f^{\frac{1}{2}}(0) \leq \frac{C_{1}}{2} \leq \Phi(0)$. And $f$ satisfies

$$
\frac{\partial}{\partial t} f \leq \triangle f+\frac{2}{R} \nabla f \cdot \nabla R-4 R f\left[f-C_{1} f^{1 / 2}+\frac{1}{n(n-1)}-c_{2} \frac{|W|}{R}\right] .
$$


Notice that $\sqrt{f(0)} \leq \frac{C_{1}}{2} \leq \Phi(0)$, then it follows from the maximum principle that $f^{\frac{1}{2}}(t) \leq \Phi(t)$. Since if $\max f^{\frac{1}{2}}(t)>\Phi(t)$, then we have

$$
\frac{d}{d t}^{+} \max f(t) \leq 0
$$

Proof. (Theorem 3.1) The inequality (3.2) now follows from standard inequalities.

As a direct consequence of Theorem 3.1, we have the following claim:

Corollary 3.1. Let $(M, g(t)), t \in[0, T)$, be an maximal solution to the Ricci flow, where $T<\infty$. Then we have

(1) either $\lim \sup _{[0, T)} R=\infty$,

(2) or limsup $\sup _{[0, T)} R<\infty$ but limsup $\sup _{[0, T)} \frac{|W|}{R} \rightarrow \infty$.

Proof. For any finite time singularity, the whole Riemannian curvature (or Ricci curvature) blows up at $T$. The Riemannian curvature tensor is decomposed into the scalar curvature part $R$, the traceless Ricci tensor $E$ and the Weyl tensor $W$. Following from Theorem 3.1, $E$ can not blow up if $R$ and $\frac{|W|}{R}$ are both bounded, hence the statement follows.

Another interesting application of (3.2) is the case when the Weyl tensor $|W|$ is controlled by the scalar curvature $R$.

Corollary 3.2. (positive isotropic curvature) Let $\left(M^{n}, g(t)\right), t \in[0, T)$, be an maximal solution to the Ricci flow, $n \geq 4$. Assuming that $g(0)$ has positive isotropic curvature, then we have $\lim \sup _{[0, T)} R=\infty$.

Proof. Since positive isotropic curvature is preserved by the Ricci flow. If dimension is larger than 4, positivity of isotropic curvature implies that the Ricci curvature is bounded by

$$
|\mathrm{Rc}|<c R
$$

for some constant $c$ only depends on the dimension $n$ (in fact, the whole Riemannian curvature tensor $|\mathrm{Rm}|$ is bounded by $R$ ). It then follows from 
Sesum's result that

$$
\lim \sup _{[0, T)} R=\infty .
$$

In dimension 4, this implies positive Weitzenböck operator. So we have

$$
P=\operatorname{Rc} \circ g-2 \mathrm{Rm}=\frac{R}{6} g \circ g-W>0,
$$

and $W$ is traceless, this implies that

$$
\frac{|W|}{R}<c_{3}
$$

Substituting this into (3.2), it follows from elementary inequalities that:

$$
\frac{|E|}{R} \leq C
$$

Since positive isotropic curvature implies scalar curvature $R>0$, the maximal existence time $T<\infty$, so

$$
\lim \sup _{[0, T)} R=\infty
$$

\section{Finite-time singularities, dilation limits and singularity models}

In this section, we will use the pinching estimate in Section 3 to study the dilation limit of Ricci flow solutions. We first need to introduce some notations. In [11, Section 16], Hamilton introduced the notion of singularity model. Roughly speaking, these are dilation limits of the Ricci flow. We briefly describe the strategy here, to find out exact details about how to dilate singularities based on rate of blowup of the curvature, see [11, Section 16] or [5, Chapter 8]. If we dilate the solution to the Ricci flow about a sequence of points and times $\left(x_{i}, t_{i}\right)$, where $x_{i} \in M$ and $t_{i} \rightarrow T$, we may choose the sequence of points and time so that $|\operatorname{Rm}|\left(x_{i}, t_{i}\right)$ is comparable to the global maximum over the space $M$ and sufficiently large previous time intervals. We now can define a sequence of pointed dilation solutions 
$\left(M, g_{i}(t), x_{i}\right)$ by:

$$
g_{i}(t)=|\operatorname{Rm}|\left(x_{i}, t_{i}\right) \cdot g\left(t_{i}+\frac{t}{|\operatorname{Rm}|\left(x_{i}, t_{i}\right)}\right),
$$

for time interval

$$
-t_{i}|\operatorname{Rm}|\left(x_{i}, t_{i}\right) \leq t<\left(T-t_{i}\right)|\operatorname{Rm}|\left(x_{i}, t_{i}\right),
$$

such that the curvature $|\operatorname{Rm}|_{g_{i}}\left(x_{i}, 0\right)=1$ and the maximum of the (Riemannian) curvature of $g_{i}$ becomes uniformly bounded for $t \leq 0$, hence we have a sequence of solutions to the Ricci flow. For finite time singularities on closed manifolds, Perelman's No Local Collapsing Theorem [22] provides the injectivity radius estimate, which is necessary to obtain a noncollapsed limit. Then we can apply Hamilton's Cheeger-Gromov type compactness theorem [10] to extract a limit solution of the Ricci flow. This is a complete solution to the Ricci flow with bounded curvature. If the solution is Type I, it is an ancient solution; if the solution is Type II, then it is an eternal solution. It is worth mentioning that in dimension 3, all dilation limits have nonnegative sectional curvature due to the pinching estimate of Hamilton [11] and Ivey [13].

Our main result in this section is the following:

Theorem 4.1. Let $(M, g(t)), t \in[0, T)$, be an maximal solution to the Ricci flow with positive scalar curvature. Then we have one of the following:

(1) either $\lim \sup _{[0, T)} R=\infty$,

(2) or if $\lim \sup _{[0, T)} R<\infty$, then $\lim \sup _{[0, T)}|W|>\frac{C}{(T-t)^{2-\delta}}$ for any constant $C>0$ and $\delta>0$. Furthermore, this must be a Type II maximal solution, and the dilation limit is a complete Ricci-flat solution with $\max |W|=1$.

Remark 4.1. Most recently, B. Wang improves this result in [28], his proof uses a different method.

We first consider Type I solutions, which has been studied extensively recently by Enders et al. [7], also by Zhang and the author [4].

Corollary 4.1. If the solution of the Ricci flow is a Type I solution, then we have $\frac{|W|}{R}$ is bounded, hence $R \rightarrow \infty$. 
Proof. This corollary essentially follows from [7, Theorem 1.8] that all Type I singularity notions are equivalent. The second part of the claim is also a direct consequence of Theorem 4.1.

Remark 4.2. From [7] and [4], such type I dilation limit must be a nontrivial gradient Ricci solitons. Notice that the dilation limit cannot be Ricci flat, otherwise this contradicts a theorem [23, Theorem 3] of Pigola et al.

We now finish our proof of Theorem 4.1.

Proof. (Theorem 4.1). We assume that $\lim \sup _{[0, T)} R<\infty$, since this is a finite time singularity, the whole Riemannian curvature tensor $|\mathrm{Rm}|$ blows up. So lim $\sup _{[0, T)}|W|=\infty$. Now if lim $\sup _{[0, T)}|W|<\frac{C}{(T-t)^{2-\delta}}$ for some constant $C>0$ and $\delta>0$, then by (3.2), the (traceless and hence) Ricci tensor is also bounded by

$$
|\mathrm{Rc}|<\frac{C}{(T-t)^{1-\delta / 2}}
$$

This implies that

$$
\int_{0}^{T}|\mathrm{Rc}| d s \leq C
$$

and a uniform bound on the distance change. Since the scalar curvature is also uniformly bounded on $[0, T)$, a similar proof as in [25] yields that the solution of the Ricci flow can be extended past $T$. This contradicts the fact that it is a finite-time maximal solution.

The rest of the theorem follows from standard blow-up analysis. Since $R$ is bounded and $\lim \sup _{[0, T)}|W|=\infty$. So the dilation limit will have $\tilde{R}=0$ and $|\tilde{W}|=1$ at the origin and time 0 . The evolution equation of scalar curvature implies that it is in fact Ricci-flat.

\section{Acknowledgment}

The author would like to thank Simon Brendle, Bennett Chow, John Lott, Maria Helena Noronha, Duong H. Phong, Harish Seshadri, Gang Tian, Hung Tran, Bing Wang and Zhou Zhang for their interests and discussions. He would also like to express his gratitude to both referees for their helpful suggestions improving this paper. Research partially supported by NSF grant DMS 0904432 and by the Jeffrey Sean Lehman Fund from Cornell University. 


\section{References}

[1] M. Berger, Les variétés Riemanniennes (1/4)-pincées, Ann. Scuola Norm. Sup. Pisa (3) 14 (1960), 161-170.

[2] S. Brendle and R. Schoen, Manifolds with 1/4-pinched curvature are space forms, J. Amer. Math. Soc. 22(1) (2009), 287-307.

[3] H.D. Cao, Deformation of Kähler metrics to Kähler-Einstein metrics on compact Kähler manifolds, Invent. Math. 81(2) (1985), 359-372.

[4] X. Cao and Q.S. Zhang, The conjugate heat equation and ancient solutions of the Ricci flow, Adv. Math. 228(5) (2011), 2891-2919.

[5] B. Chow and D. Knopf, The Ricci flow: an introduction, Math. Surveys Monogr. 110, American Mathematical Society, Providence, RI, 2004.

[6] D.M. DeTurck, Deforming metrics in the direction of their Ricci tensors, J. Diff. Geom. 18(1) (1983), 157-162.

[7] J. Enders, R. Müller and P.M. Topping, On Type I singularities in Ricci flow, (2010), arXiv: 1005.1624v2.

[8] R.S. Hamilton, Three-manifolds with positive Ricci curvature, J. Diff. Geom. 17(2) (1982), 255-306.

[9] R.S. Hamilton, Four-manifolds with positive curvature operator, J. Diff. Geom. 24(2) (1986), 153-179.

[10] R.S. Hamilton, A compactness property for solutions of the Ricci flow, Amer. J. Math. 117(3) (1995), 545-572.

[11] R.S. Hamilton, The formation of singularities in the Ricci flow, in 'Surveys in Differential Geometry', II (Cambridge, MA, 1993), International Press, Cambridge, MA, 1995, 7-136.

[12] R.S. Hamilton, Four-manifolds with positive isotropic curvature, Comm. Anal. Geom. 5(1) (1997), 1-92.

[13] T. Ivey, Ricci solitons on compact three-manifolds, Diff. Geom. Appl. 3(4) (1993), 301-307.

[14] W. Klingenberg, Über Riemannsche Mannigfaltigkeiten mit positiver Krümmung, Comment. Math. Helv. 35 (1961), 47-54. 
[15] D. Knopf, Estimating the trace-free Ricci tensor in Ricci flow, Proc. Amer. Math. Soc. 137(9) (2009), 3099-3103.

[16] L. Ma and L. Cheng, On the conditions to control curvature tensors of Ricci flow, Ann. Global Anal. Geom. 37(4) (2010), 403-411.

[17] M.J. Micallef and J.D. Moore, Minimal two-spheres and the topology of manifolds with positive curvature on totally isotropic two-planes, Ann. Math. (2) 127(1) (1988), 199-227.

[18] M.J. Micallef and McKenzie Y. Wang, Metrics with nonnegative isotropic curvature, Duke Math. J. 72(3) (1993), 649-672.

[19] H.T. Nguyen, Isotropic curvature and the Ricci flow, Int. Math. Res. Not. IMRN, 2010(3) (2010), 536-558.

[20] M.H. Noronha, Self-duality and 4-manifolds with nonnegative curvature on totally isotropic 2-planes, Michigan Math. J. 41(1) (1994), 3-12.

[21] M.H. Noronha, Positively curved 4-manifolds and the nonnegativity of isotropic curvatures, Michigan Math. J. 44(2) (1997), 211-229.

[22] G. Perelman, The entropy formula for the Ricci flow and its geometric applications, (2002), arXiv:math.DG/0211159.

[23] S. Pigola, M. Rimoldi and A.G. Setti, Remarks on non-compact gradient Ricci solitons, Math. Z. 268(3-4) (2011), 777-790.

[24] W. Seaman, On manifolds with nonnegative curvature on totally isotropic 2-planes, Trans. Amer. Math. Soc. 338(2) (1993), 843-855.

[25] N. Šešum, Curvature tensor under the Ricci flow, Amer. J. Math. 127(6) (2005), 1315-1324.

[26] N. Sesum and G. Tian, Bounding scalar curvature and diameter along the Kähler Ricci flow (after Perelman), J. Inst. Math. Jussieu 7(3) (2008), 575-587.

[27] B. Wang, On the conditions to extend Ricci flow, Int. Math. Res. Not. IMRN 2008(8) (2008), (Art. ID rnn012, 30).

[28] B. Wang, On the conditions to extend Ricci flow (ii), July 2011, arXiv: $1107.5107 \mathrm{v} 1$.

[29] R. Ye, Curvature estimates for the Ricci flow. II, Calc. Var. Partial Differ. Equ. 31(4) (2008), 439-455. 
[30] Z. Zhang, Scalar curvature behavior for finite-time singularity of Kähler-Ricci flow, Michigan Math. J. 59(2) (2010), 419-433.

Department of Mathematics

Cornell University

ITHACA, NY 14853-4201

USA

E-mail address: cao@math.cornell.edu

ReCeived January 18, 2011 